\title{
Effect of Splenectomy and Autologous Spleen Transplantation on the Serum Platelet-Activating Factor Acetylhydrolase (PAF-AH) Activity and Acute Phase Response (APR) in a Porcine Model
}

\author{
Romana Turk, ${ }^{1,7}$ Dražen Vnuk, ${ }^{2}$ Ante Svetina, ${ }^{1}$ Zlata Flegar-Meštrić, ${ }^{3}$ Mirna Robić, ${ }^{1}$ \\ Nenad Turk, ${ }^{4}$ Vilim Starešina, ${ }^{4}$ Vlatko Rumenjak, ${ }^{5}$ and Dubravka Juretićc
}

\section{Erratum to: Inflammation}

DOI 10.1007/s10753-009-9117-3

The original version of this article unfortunately contained a mistake. Zlata Flegar-Meštrić, Mirna Robić and Dubravka Juretić were not listed correctly in the online version of this article.

The online version of the original article can be found at http://dx.doi. org/10.1007/s10753-009-9117-3

\footnotetext{
${ }^{1}$ Department of Pathophysiology, Faculty of Veterinary Medicine, University of Zagreb, Heinzelova 55, 10000 Zagreb, Croatia

${ }^{2}$ Surgery, Orthopedics and Ophthalmology Clinic,

Faculty of Veterinary Medicine, University of Zagreb, Zagreb, Croatia

${ }^{3}$ Institute of Clinical Chemistry, Clinical Hospital "Merkur", Zagreb, Croatia

${ }^{4}$ Department of Microbiology and Infectious Diseases with Clinic, Faculty of Veterinary Medicine, University of Zagreb, 10000 Zagreb, Croatia

${ }^{5}$ Department of Medical Laboratory Diagnostics, Sveti Duh General Hospital, Zagreb, Croatia

${ }^{6}$ Department of Medical Biochemistry and Hematology, Faculty of Pharmacy and Biochemistry, University of Zagreb, Zagreb, Croatia

${ }^{7}$ To whom correspondence should be addressed at Department of Pathophysiology, Faculty of Veterinary Medicine, University of Zagreb, Heinzelova 55, 10000 Zagreb, Croatia. E-mail: rturk@vef.hr
} 